\title{
Asymptomatic Portal Cavernoma Revealing Activated Protein C Resistance
}

\author{
Salem Bouomrani*, Nesrine Regaïeg, Mayada BenHamad, Souad Yahyaoui and Nesrine Belgacem \\ Department of Internal medicine, Military Hospital of Gabes, Tunisia
}

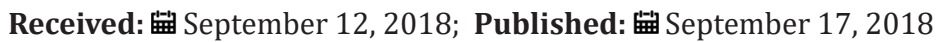

*Corresponding author: Salem Bouomrani, Department of Internal medicine, Military Hospital of Gabes, Gabes 6000, Tunisia

\begin{abstract}
Introduction: Unlike the child, the portal cavernoma (PC) remains an exceptional and unusual complication of portal thrombosis in adults and the elderly. We report an original observation of asymptomatic PC secondary to chronic portal thrombosis revealing Factor V Leiden mutation in a young adult.
\end{abstract}

Observation: A 27-year-old female with no significant pathological history was explored for thrombocytopenia found on a routine check-up. Radiological investigation concluded to the diagnosis of post-thrombotic PC with extra-hepatic portal hypertension. Systematic screening for acquired and inherited thrombophilia objectified activated protein $\mathrm{C}$ resistance, and genetic test confirmed the specific mutation of the coagulation factor V gene (Factor V Leiden). Under effective anticoagulation, the evolution was favorable.

Conclusion: As rare as it is in adult, PC deserves to be known and his diagnosis imposes a systemic screening for inherited thrombophilic disorders even in the absence of family and/or personal history of thromboembolic disease.

Keywords: Portal Cavernoma; Portal Vein Thrombosis; Factor V Leiden; Activated Protein C Resistance; Inherited Thrombophili

\section{Introduction}

Relatively recently known as a clinical entity, portal cavernoma (PC) is poorly known by clinicians; his first description was autopsy in 1903 [1] and clinical in 1944 [2]. It is defined as the replacement of the portal vein by a network of collateral veins and venules, tortuous and hepatopetous, giving the appearance of a vascular bundle in the hepatic hilus $[3,4]$. The causes are occlusion or often chronic thrombosis of the extrahepatic portal system [35]. The PC represents a real diagnostic challenge because it often remains asymptomatic and would be fortuitous discovery on abdominal radiological explorations [3-5]. More rarely, the clinic may be growth retardation in children, upper or lower digestive bleeding, or feeling of abdominal heaviness. These signs are the consequences of extrahepatic portal hypertension secondary to PC [3-5]. Ultrasound, Doppler examination, computed tomography and magnetic resonance imaging (MRI) allow positive diagnosis of PC and detection of its complications [3-5]. PCs following chronic thrombosis of the portal vein are rare in adults [6]. This thrombosis may rarely be due to hereditary thrombophilia type activated protein C resistance (APCR) [7-10]; indeed, only one case of APCR was found as the etiology of this thrombosis in the 36-case series of Denninger MH et al. [7]. The APCR is a hereditary state of blood hypercoagulability, secondary to a point mutation of the coagulation factor V gene [11]. This mutation (R506Q), giving the mutated factor called factor $\mathrm{V}$ Leiden, confer resistance to the action of activated protein $\mathrm{C}$ and thus increases the risk of thromboembolism [11]. We report an original observation of PC secondary to chronic portal thrombosis revealing APCR/Factor V Leiden in a young adult.

\section{Case Report}

A 27-year-old female with no significant pathological history was explored for thrombocytopenia at $104000 / \mathrm{mm}^{3}$ then at $108000 / \mathrm{mm} 3$ found on a complete blood cell count requested during a routine check-up. The somatic examination was without abnormalities. Biology confirmed isolated thrombocytopenia (without abnormalities of other blood lines): platelets at 106000 / $\mathrm{mm} 3$, hemoglobin at $14 \mathrm{~g} / \mathrm{dl}$, leukocytes at $8680 / \mathrm{mm}^{3}$, neutrophils at $5550 / \mathrm{mm}^{3}$ and lymphocytes at $2520 / \mathrm{mm} 3$. In addition, there was a prothrombin level of $65 \%$ without other liver test abnormalities: total bilirubin at $14 \mu \mathrm{mol} / \mathrm{l}$, conjugate bilirubin at $2.2 \mu \mathrm{mol} / \mathrm{l}$, aspartate aminotransferase at $38 \mathrm{IU} / \mathrm{l}$, alanine transaminase at $35 \mathrm{IU} / \mathrm{l}$, alkaline phosphatase at $184 \mathrm{IU} / \mathrm{l}$, gammaglutamyl transpeptidase at $11 \mathrm{IU} / \mathrm{l}$ and vitamin $\mathrm{K}$ at $324 \mathrm{ng} / \mathrm{l}$. The rest of the basic biology was within normal limits (glycemia, serum 
calcium, ionogram, creatinine, muscle enzymes, total cholesterol, triglycerides, erythrocyte sedimentation rate, C-reactive protein, and electrophoresis of serum proteins). The abdominal ultrasound with Doppler examination showed a moderate and homogeneous splenomegaly, and the absence of visualization of the portal vein which is replaced by a tubular, tortuous, and trans-sound structures evoking a PC (Figure 1).

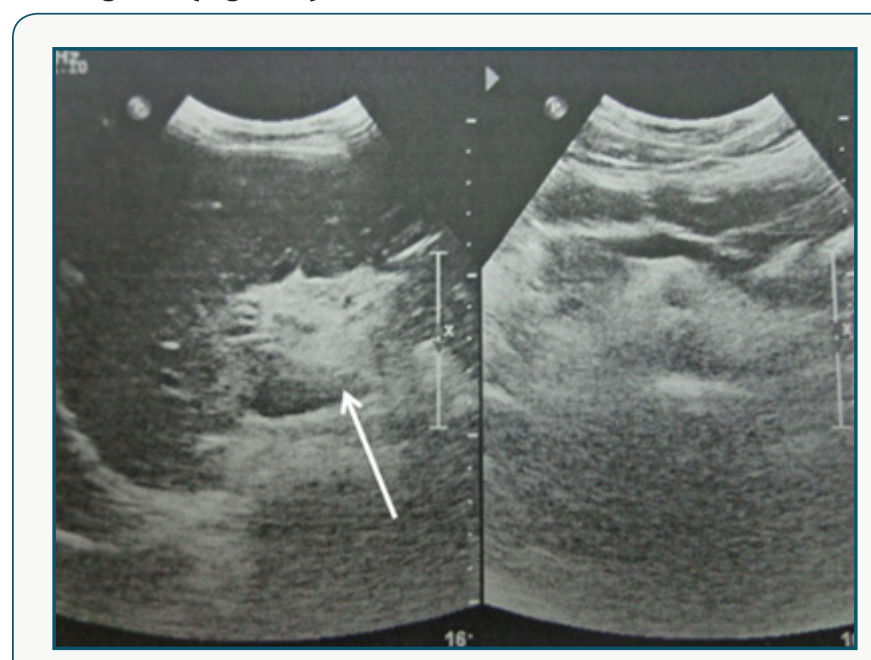

Figure 1: Abdominal ultrasonography with Doppler examination: absence of the portal vein which is replaced by a cluster of small veins and venules in the hepatic hil (arrow).

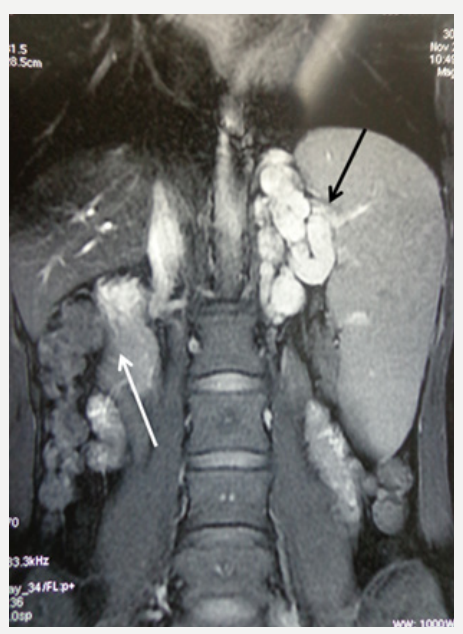

Figure 2: Coronal T1-weighted abdominal MRI with gadolinium: Portal Cavernoma (white arrow) and splenomegaly with very marked splenorenal collateral circulation (black arrow).

MRI of the abdomen confirmed the diagnosis of PC, probably related to chronic thrombosis of the portal vein (Figure 2) with marked splenorenal collateral venous circulation (Figures 2 \& 3 ), and moderate splenomegaly at $18 \mathrm{~cm}$ without focal lesions (Figures 2 \& 3). Oeso-gastroduodenal fibroscopy noted the presence of grade I oesophageal varices. Thus the diagnosis of a PC with extrahepatic portal hypertension was retained. Given the absence of known local, locoregional or systemic causes that could explain portal thrombosis, a systematic screening for acquired and inherited thrombophilia was requested. Anti-nuclear antibodies, anti-soluble nuclear antigens antibodies, anti-neutrophil cytoplasm antibodies, anti-phospholipids antibodies, lupus anticoagulant, and cryoglobulins were negative. Activated protein $\mathrm{C}$ was at $65 \%$, activated protein $\mathrm{S}$ at $70 \%$, and antithrombin III at $83 \%$. The search for APCR with coagulometry was positive: Coagulation time without activated protein $\mathrm{C}$ at 43 seconds and coagulation time with activated protein $\mathrm{C}$ at 300 seconds. The specific factor $\mathrm{V}$ mutation (G1691A) was subsequently confirmed by the genetic test. The patient was effectively anticoagulated with a favorable evolution.

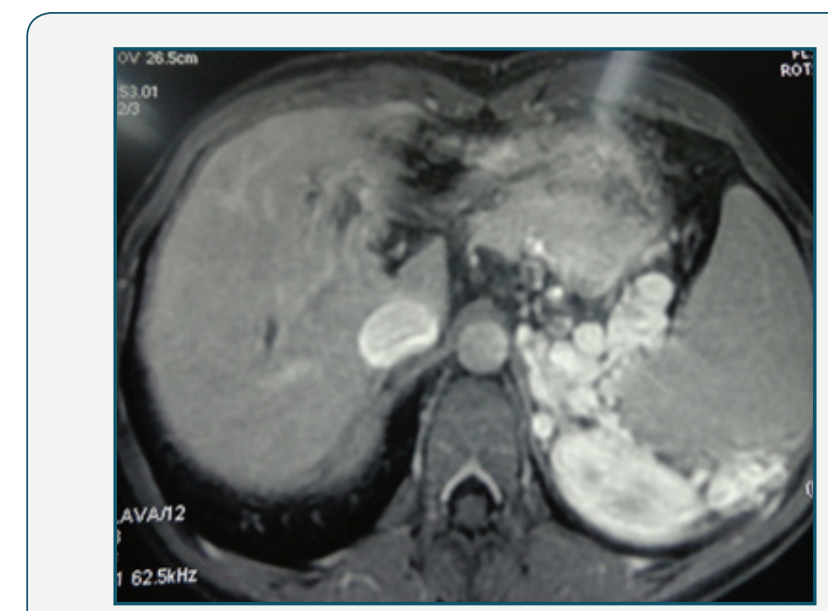

Figure 3: Axial T1-weighted abdominal MRI with gadolinium: splenomegaly with very marked splenorenal collateral circulation.

\section{Discussion}

Thrombosis of the portal vein is rare in adults and in the Western world [3]: The large Swedish autopsy series of 23796 cases concluded with a prevalence of portal thrombosis of $1 \%$ in the general population [12]. The pathogenesis of these thromboses is classically multifactorial resulting from the combination of local causes and systemic prothrombotic conditions $[3,4]$. The main local causes are abdominal trauma, local infections, abdominal surgery, inflammatory bowel diseases, colon diverticulosis, appendicitis, chronic liver diseases with or without cirrhosis, and digestive cancers $[3,5,13]$. The prothrombotic diseases most frequently responsible for portal thromboses are myeloproliferative syndromes, paroxysmal nocturnal hemoglobinuria, antiphospholipid syndrome, and hereditary thrombophilias such as deficiency of protein C, S, and antithrombin III, factor V Leiden, and mutation of the factor II gene [3-5]. The portal cavernoma typically complicates chronic portal thrombosis and child's portal thrombosis [3-5]. The coagulation factor $\mathrm{V}$ mutation (factor $\mathrm{V}$ Leiden) responsible for APCR is rarely reported as etiology of portal thrombosis; its frequency is estimated at $2.8-7.6 \%$ in the different series [7-10]. It is likewise exceptionally reported as the cause of portal cavernomas [6]. However, these clinical findings seem to be very underestimated: indeed, the systematic screening noted the factor $\mathrm{V}$ Leiden mutation in $30 \%$ of cases of child portal 
thrombosis in the series of El-Karaksy H et al. [14]. Similarly, in the series of Egesel T et al. [15], of 23 patients with portal cavernoma initially labeled "idiopathic", the systematic complete screening for inherited thrombophilias revealed APCR in $30 \%$ of cases [15].

Thus, both APCR and factor V Leiden mutation appear to be significantly associated with portal thrombosis and cavernomatous transformation of this vein $[8,15]$; this was confirmed by the extensive review of the literature by Qi X et al. [16], where factor $\mathrm{V}$ Leiden mutation was significantly associated with portal thrombosis with or without cirrhosis, with an odds ratio of 1.85 and 2.55 respectively [16]. Also note that several inherited thrombophilic disorders may associate to give portal thrombosis and portal cavernoma in the same patient [7].

The treatment of PCsecondary to APCR/factorVLeiden mutation is mainly effective anticoagulation for life, which will mainly allow to limit the extension of thrombosis, thrombotic recurrence, and PC-specific ischemic mesenteric complications [3-5]. Surgical treatment such as portal decompression with portosystemic shunt is indicated in PC cases complicated by hepatobiliary involvement ("portal biliopathy" or "Portal cavernoma cholangiopathy") [3,5].

\section{Conclusion}

Unlike the child, the portal cavernoma remains an exceptional and unusual complication of portal thrombosis in adults and the elderly. His diagnosis imposes a systemic screening for inherited thrombophilic disorders even in the absence of family and/or personal history of thromboembolic disease. Only early diagnosis and timely and adapted care are the guarantors of a good evolution. The lack of knowledge of this pathology as well as the resulting diagnostic and/or therapeutic delay make the prognosis of the PC very pejorative.

\section{References}

1. Gibson J, Richards R (1955) Cavernous transformation of the portal vein. J Pathol Bact 70: 81-95.

2. Fraser J, Brown AK (1944) A clinical syndrome associated with a rare anomaly of the vena portae system. Surg Gynecol Obstet 78: 520-524.

3. Condat B (2006) Portal vein thrombosis. Gastroenterol Clin Biol 30(10): $1170-1176$.

This work is licensed under Creative Commons Attribution 4.0 License

To Submit Your Article Click Here: Submit Article

DOI: $10.32474 / \mathrm{CTGH.2018.01.000116}$
4. Kumar A, Sharma P, Arora A (2015) Review article: Portal vein obstruction--epidemiology, pathogenesis, natural history, prognosis and treatment. Aliment Pharmacol Ther 41(3): 276-292.

5. Vibert E, Azoulay D, Castaing D, Bismuth H (2002) Portal cavenorma: Diagnosis, aetiologies and consequences. Ann Chir 127(10): 745-750.

6. Georgescu E, Dumitrescu D, Ionescu R (2010) Portal cavernoma in a patient with Crohn's disease associated with factor $\mathrm{V}$ Leiden mutation and antiphospholipid syndrome. J Gastrointestin Liver Dis 19(4): 449452.

7. Denninger MH, Chaït Y, Casadevall N, Hillaire S, Guillin MC, et al. (2000) Cause of portal or hepatic venous thrombosis in adults: The role of multiple concurrent factors. Hepatology 31(3): 587-591.

8. Janssen HL, Meinardi JR, Vleggaar FP, van Uum SH, Haagsma EB, et al. (2000) Factor V Leiden mutation, prothrombin gene mutation, and deficiencies in coagulation inhibitors associated with Budd-Chiari syndrome and portal vein thrombosis: Results of a case-control study. Blood 96(7): 2364-2368.

9. Bhattacharyya M, Makharia G, Kannan M, Ahmed RP, Gupta PK, et al. (2004) Inherited prothrombotic defects in Budd-Chiari syndrome and portal vein thrombosis: A study from North India. Am J Clin Pathol 121(6): 844-847.

10. Primignani M, Martinelli I, Bucciarelli P, Battaglioli T, Reati R, et al. (2005) Risk factors for thrombophilia in extrahepatic portal vein obstruction. Hepatology 41(3): 603-608.

11. Kujovich JL (2011) Factor V Leiden thrombophilia. Genet Med 13(1): $1-16$.

12. Ogren M, Bergqvist D, Björck M, Acosta S, Eriksson H, et al. (2006) Portal vein thrombosis: Prevalence, patient characteristics and lifetime risk: A population study based on 23,796 consecutive autopsies. World J Gastroenterol 12(13): 2115-2119.

13. Salem B, Afef F, Nadia B, Nabil A, Maher B (2013) Portal vein thrombosis in a type 1 autoimmune hepatitis. Pan Afr Med J 14: 130.

14. El Karaksy H, El Koofy N, El Hawary M, Mostafa A, Aziz M, et al. (2004) Prevalence of factor V Leiden mutation and other hereditary thrombophilic factors in Egyptian children with portal vein thrombosis: Results of a single-center case-control study. Ann Hematol 83(11): 712715 .

15. Egesel T, Büyükasik Y, Dündar SV, Gürgey A, Kirazli S, et al. (2000) The role of natural anticoagulant deficiencies and factor $V$ Leiden in the development of idiopathic portal vein thrombosis. J Clin Gastroenterol 30(1): 66-71.

16. Qi X, Ren W, De Stefano V, Fan D (2014) Associations of coagulation factor V Leiden and prothrombin G20210A mutations with Budd-Chiari syndrome and portal vein thrombosis: A systematic review and metaanalysis. Clin Gastroenterol Hepatol 12(11): 1801-1812.

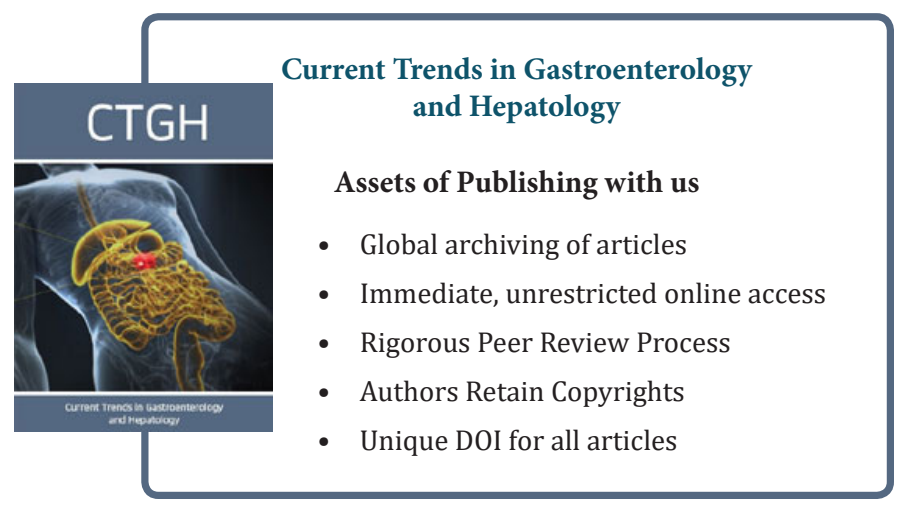

